\title{
Structural and Compositional Analysis of Core/Shell QDs by Transmission Electron Microscopy Techniques
}

\author{
N. Fernández-Delgado ${ }^{1}$, M. Herrera ${ }^{1}$, J. Pizarro ${ }^{2}$, P. L. Galindo ${ }^{2}$, P.J. Rodriguez-Cantó ${ }^{3}$, R. Abargues ${ }^{3,4}$, \\ J.P. Martínez-Pastor ${ }^{4}$, S. I. Molina ${ }^{1}$ \\ 1. Department of Material Science, Metallurgical Engineering and Inorganic Chemistry, IMEYMAT, \\ Faculty of Science, University of Cadiz, 11510 Puerto Real, Cadiz, Spain. \\ 2. Department of Computer Engineering, School of Engineering, University of Cadiz, Avda. de la \\ Universidad de Cádiz nº10, 11519, Puerto Real, Cadiz, Spain. \\ 3. INTENANOMAT S.L., C/Catedrático José Beltrán 2, 46980 Paterna, Spain. \\ 4. Instituto Ciencia de los Materiales, University of Valencia, P.O. Box 22085, 46071 Valencia, Spain.
}

Colloidal QDs have drawn the attention of researches because of their remarkable properties, such as quantum dot confinement and their low cost synthesis method. They are being used in a wide range of applications such as multiplexed biological detection [1] or ultrahigh-efficiency solar photon conversion [2]. Improvements in the optoelectronic properties of the colloidal QDs have been observed when they are covered with a shell [3]. It has the function of avoiding re-absorption of the light emitted by the core of the QD [4]. In particular, CdSe/ZnS QDs are being extensively used in the photovoltaic field [5]. In this communication, we have analyzed structural and compositional properties of core/shell QDs through Transmission Electron Microscopy techniques, in order to understand the performance of the core and the shell and correlate this behavior with their optoelectronic properties. We have studied two core/shell QDs with a core of CdSe and a shell of $\mathrm{ZnS}$. The core has a nominal diameter of $2.7 \mathrm{~nm}$ and a nominal shell of $0.7 \mathrm{~nm}$. The samples were prepared for the study by dipping a holey carbon grid in the solution with the core/shell QDs. Fig. 1 shows a High Resolution TEM image acquired at $200 \mathrm{kV}$ where some $\mathrm{CdSe} / \mathrm{ZnS}$ QDs can be observed; one of them has been marked at the image. The average diameter is $3.5 \pm 1.1 \mathrm{~nm}$ and the morphology seems to be uniform, in concordance with the expected value. However, different crystalline structures are not found in the QDs, and thus the shell cannot be distinguished from the core by this technique. One likely reason may be that despite the high lattice mismatch between the core and the shell, $12.4 \%$, the small size of the shell allows it to grow coherently around the core. Moreover, the QDs seem to be affected by the electron beam. The working conditions are being optimized in order get a more detailed study of the samples without damaging the QDs. An alternative technique in order to distinguish between the core and the shell and study their interface is High Angle Annular Dark Field Scanning TEM (HAADF-STEM). Fig. 2 shows a HAADF-STEM image acquired at $200 \mathrm{kV}$ of the $\mathrm{CdSe} / \mathrm{ZnS}$ QDs. In this technique, the intensity of the image is strongly related to the atomic number. It can be observed that even though at the edge of the QDs the intensity is lower, as expected from the lower atomic numbers of the shell components, the amount of material in that area is lower too. Also, the solution in which the QDs are diluted contains organic compounds from the colloidal synthesis that somehow interferes in the HAADF-STEM analysis. All these facts provoke a more complex analysis of the intensity. HAADF-STEM image simulations are being carried out in order to make the interpretation of the images easier. First simulation results have shown that the shell may produce a compression of the core. The consequences of this hypothetical compression in the QDs are being studied in detail. In addition, knowing about the difficulty of this work and in order to add information to the obtained results, compositional analyses are being carried out by Energy Dispersive X-Ray (EDX). The experimental conditions for this study are being optimized because of the small size of the nanoparticles that make them be unstable under the beam [6]. 
References:

[1] W.C.W. Chan et al., Current Opinion in Biotechnology 13 (2002), p. 46.

[2] A.J. Nozik, Inorganic Chemistry 44 (2005), p. 6893.

[3] H.J. Byun, et al., Nanotechnology, 22 (2011), p. 235605.

[4] F. Meinardi, et al., Nature Photonics 8 (2014), p. 392.

[5] A.J. Nozik, Physica E 14 (2002), p. 115.

[6] Acknowledgements: This work was supported by the Spanish MINECO (projects TEC2014-53727C2-1-R, -2-R and CONSOLIDER INGENIO 2010 CSD2009-00013) and Junta de Andalucía (PAI research group TEP-946). The research leading to these results has received co-funding from the European Union.



Figure 1. HRTEM image acquired at $200 \mathrm{kV}$ of CdSe/ZnS QDs.



Figure 2. HAADF-STEM image acquired at $200 \mathrm{kV}$ of CdSe/ZnS QDs. 